\title{
Análise faunística das formigas epígeas (Hymenoptera, Formicidae) em campo nativo no Planalto das Araucárias, Rio Grande do Sul
}

\author{
Emília Zoppas de Albuquerque ${ }^{1,2} \&$ Elena Diehl ${ }^{1,3}$
}

${ }^{1}$ Programa de Pós-Graduação em Biologia: Diversidade e Manejo de Vida Silvestre, Laboratório de Insetos Sociais, Universidade do Vale do Rio dos Sinos. Caixa Postal 275, 93001-970 São Leopoldo-RS, Brasil.

${ }^{2}$ Bolsista CAPES/PROSUP. emilia_albuq@ hotmail.com

${ }^{3}$ Pesquisadora CNPq. elena.diehl@ pq.cnpq.br

\begin{abstract}
Faunal analysis of epigaeic ants (Hymenoptera, Formicidae) in native fields of the Planalto das Araucárias, State of Rio Grande do Sul. This paper describes the richness, composition and occurrence patterns of the epigaeic ants in native field areas. Eight field plots were sampled, in which two $100 \mathrm{~m}$ transects, distanced $50 \mathrm{~m}$ from each other, were traced. Along those transects, at every $10 \mathrm{~m}$, pitfalls and sardine baits at intervals of two meters, were installed totalizing 20 pitfalls and 20 baits per plot. Additional collecting was performed by manual ant capture on the ground. In total 32 species of epigaeic ants were collected, distributed in 16 genera, 12 tribes and five subfamilies. By comparing the observed richness with the estimated richness for pitfalls as well as for baits, the numbers of collected species represented, respectively, $79.4 \%$ and $69.4 \%$ of the total estimated community. One species of the Formicinae subfamily, Acropyga goeldii, has been recorded for the first time in Rio Grande do Sul.
\end{abstract}

KEYWORDS. Acropyga goeldii; ant fauna; epigaeic ants; inventory.

RESUMO. Análise faunística das formigas epígeas (Hymenoptera, Formicidae) em campo nativo na região do Planalto das Araucárias, Rio Grande do Sul. Este estudo descreve a riqueza, a composição e o padrão de ocorrência de formigas epígeas em ambiente de campo nativo. As coletas foram realizadas em oito parcelas de campo, nas quais foram traçados dois transectos de $100 \mathrm{~m}$, espaçados entre si cerca de $50 \mathrm{~m}$. Ao longo dos transectos, a cada dez metros, foram instaladas armadilhas de solo e iscas de sardinha, a intervalos de dois metros, totalizando 20 iscas e 20 armadilhas por parcela. Coletas adicionais foram realizadas por captura manual das formigas encontradas no solo. No total foram coletadas 32 espécies de formigas epígeas, distribuídas em 16 gêneros, 12 tribos e cinco subfamílias. Comparando-se as riquezas observadas com os valores da riqueza estimada, tanto para as armadilhas como para as iscas, os números de espécies coletadas representaram, respectivamente, $79,4 \%$ e $69,4 \%$ da comunidade total estimada. Uma espécie da subfamília Formicinae, Acropyga goeldii foi registrada pela primeira vez para o Rio Grande do Sul

PALAVRAS-CHAVE. Acropyga goeldii; formigas epigéicas; inventário; mirmecofauna.

As formigas apresentam ampla distribuição geográfica (Hölldobler \& Wilson 1990; Jaffé 1993), constituindo cerca de $1,5 \%$ da fauna global de insetos (Wilson 2000) e aproximadamente $10 \%$ da biomassa animal nas florestas tropicais (Fitkau \& Klinge 1973), campos e, provavelmente, nos principais ecossistemas do mundo. Mais de 11.000 espécies de formigas já foram descritas, mas as estimativas mostram que esse número pode superar 21.800 espécies (Agosti \& Johnson 2003).

A riqueza e a composição de espécies de formigas variam de acordo com a altitude, latitude e condições climáticas como temperatura e umidade. Segundo Kusnezov (1957), a riqueza de espécies de formigas tende a diminuir com o aumento da latitude, com o aumento da altitude em relação ao nível do mar e com o aumento da aridez do solo. Parece existir uma relação positiva entre riqueza de espécies de formigas e latitude quando essa for menor de $30^{\circ}$ e uma relação negativa para altitudes iguais ou superiores a $500 \mathrm{~m}$ (Ward 2000).

As formigas, como táxon, são termofílicas, sendo raras as espécies ativas sob baixas temperaturas (Kaspari 2003). Temperaturas em torno de $30^{\circ} \mathrm{C}$, como as dos trópicos, são mais favoráveis às atividades e processos fisiológicos realizados pelas formigas (Hölldobler \& Wilson 1990), o que faz com que a região Neotropical tenha a segunda maior riqueza de gêneros, representando cerca de $39 \%$ da fauna mundial. Além disso, quando comparado com as demais regiões, o neotrópico tem a maior proporção de gêneros endêmicos (Bolton 1994; Lattke 2003).

A riqueza de espécies de formigas também pode ser influenciada por características do habitat, como a estrutura da vegetação. Um habitat com maior complexidade vegetacional fornece maior disponibilidade de locais para nidificação e maior oferta de alimento. Em florestas tropicais, a riqueza de espécies é maior do que em ambientes com formações vegetais mais homogêneas, como áreas cultivadas, campos, dunas e restingas (Benson \& Harada 1988; Bonnet \& Lopes 1993; Matos et al. 1994; Oliveira et al. 1996; Tavares et al. 2001). Nos ambientes temperados, como a variação nas formações vegetais não é tão pronunciada, o número de espécies é mais uniforme. Além disso, ambientes homogêneos parecem ter o predomínio de uma ou poucas espécies enquanto que em ambientes heterogêneos, a dominância relativa das espécies é baixa (Fowler et al. 1991; Leal \& Lopes, 1992).

Com a crescente destruição dos habitats, torna-se cada 
vez mais importante a realização de estudos sobre a diversidade biológica e de ecossistemas (Wilson 2000). O inventário de espécies pode fornecer informações sobre a distribuição zoogeográfica, propriedades biológicas e sobre a presença de espécies raras ou ecologicamente importantes, sejam elas introduzidas ou endêmicas (Wilson 1997; Alonso \& Agosti 2000). Por suas características biológicas e ecológicas (Majer 1983, 1984; Majer et al. 1984; Andersen 1987; Lobry de Bruyn 1999), o conhecimento da diversidade de formigas de uma determinada área pode fornecer importantes informações para planos de manejo e conservação, uma vez que a riqueza e a composição das espécies podem indicar o grau de conservação ou de degradação local, ou ainda uma possível vulnerabilidade a mudanças ambientais (Alonso \& Agosti 2000).

A região do Planalto das Araucárias no sul do Brasil se caracteriza pelo terreno ondulado a fortemente ondulado. Apresenta formações campestres com fisionomias distintas, como campos secos ou limpos (sendo estes os maiores em extensão), campos rupestres e campos turfosos. São constituídos predominantemente por Andropogon lateralis Nees 1829 (Gramineae), que pode atingir até $80 \mathrm{~cm}$ de altura, além de espécies cespitosas e rizomatosas de Asteraceae, Leguminosae, Poaceae e Verbenaceae (Teixeira et al. 1986; Bristot 2001). Nestas áreas de campo encontram-se distribuídos de forma irregular exemplares de Araucaria angustifolia (Bertol.) Kuntze 1893, juntamente com capões e florestas-de-galeria, cuja composição florística se assemelha à da floresta ombrófila mista (Rambo 1956; Teixeira et al. 1986; Bristot 2001).

O Planalto das Araucárias em função das suas diferentes fisionomias paisagísticas (campos, florestas e cânions) abriga uma diversidade da flora e da fauna característica da região, porém pouco ainda é conhecido sobre sua fauna de insetos epígeos. Deve ser destacado que alterações substanciais na biodiversidade local, certamente, tendem a serem maximizadas, uma vez que os campos nativos vêm sofrendo uma forte pressão antrópica decorrentes das atividades agrosilvopastoris nos últimos anos. Assim, este estudo foi realizado com o objetivo de conhecer a riqueza, a composição da mirmecofauna local, bem como identificar os padrões de ocorrência das espécies de formigas de solo em ambiente de campo nativo.

\section{MATERIALE MÉTODOS}

Área de estudo

O levantamento da mirmecofauna epígea foi realizado de agosto a dezembro de 2004, em áreas de campo nativo no município de Cambará do Sul na região do Planalto das Araucárias, em altitudes variando de 900 a 1.200 m. O clima da região é subtropical (cfa) segundo a classificação de Köppen, aproximando-se do temperado com estações perfeitamente definidas, ocorrendo intensas geadas e até neve no inverno. A temperatura média anual é de $18^{\circ} \mathrm{C}$, com mínima de $-3^{\circ} \mathrm{C}$ no inverno e máxima de $27^{\circ} \mathrm{C}$ no verão. Os índices pluviométricos variam de 1.500 a $1.750 \mathrm{~mm}$ nas partes mais baixas e de $1.750 \mathrm{a}$
$2.250 \mathrm{~mm}$ no planalto e encosta da Serra. Os nevoeiros são freqüentes o ano todo devido à condensação do vapor de água na atmosfera e pelo ar quente e úmido vindo do mar (Plano de Manejo do Parque Nacional dos Aparados da Serra 2003).

\section{Coletas}

As formigas foram coletadas em oito parcelas de campo, cada uma com 1 ha (100 m x $100 \mathrm{~m})$, das quais quatro são situadas no interior do Parque Nacional dos Aparados da Serra - PNAS ( $\left.29^{\circ} 10^{\prime} \mathrm{S} ; 50^{\circ} 05^{\prime} \mathrm{W}\right)$ e quatro são distantes do parque 9,7 km em linha reta $\left(29^{\circ} 10^{\prime} \mathrm{S} ; 50^{\circ} 11^{\prime} \mathrm{W}\right)$.

Por parcela, foram traçados dois transectos de $100 \mathrm{~m}$, distantes um do outro $50 \mathrm{~m}$, ao longo dos quais, a cada $10 \mathrm{~m} \mathrm{e}$ foram instaladas armadilhas tipo "pitfall" e iscas de sardinha a intervalos de $2 \mathrm{~m}$, totalizando 20 iscas e 20 armadilhas por parcela. As armadilhas consistiram de copos plásticos $(\phi=6,5$ $\mathrm{cm}$ ) de $200 \mathrm{~mL}$, contendo $80 \mathrm{~mL}$ de álcool $80 \%$, enterrados até a borda superior, e que permaneceram no solo por 24 horas. Como iscas foram usadas porções de cerca de $1 \mathrm{~cm}^{3}$ de sardinha em óleo vegetal dispostas sobre papel filtro $\left(9 \mathrm{~cm}^{2}\right)$ que foram recolhidas duas horas após a sua colocação. Coletas adicionais foram realizadas por captura manual das formigas encontradas no solo, tendo sido empregado um esforço amostral de uma hora por parcela (vide Romero \& Jaffé 1989; Bestelmeyer et al. 2000; Delabie et al. 2000, para eficácia e viabilidade das técnicas).

\section{Identificação}

A identificação em nível de gênero foi feita com o auxílio da chave dicotômica de Bolton (1994), adotando-se a proposta de Bolton (2003) para as subfamílias. As espécies e morfoespécies foram identificadas por comparação com a Coleção de Formicidae do Laboratório de Insetos Sociais da UNISINOS (São Leopoldo, RS), onde o material testemunho está depositado.

\section{Análise faunística}

A riqueza observada de espécies $\left(\mathrm{S}_{\mathrm{obs}}\right)$ foi obtida pelo somatório do número das espécies coletadas pelas três técnicas. A riqueza estimada $\left(\mathrm{S}_{\text {est }}\right.$ ) foi avaliada pelo estimador de riqueza jackknife de primeira ordem, utilizado separadamente para os dados das iscas de sardinha e armadilhas pelo programa EstimateS (Colwell 1997). O padrão de ocorrência (PO) foi calculado a partir do número de registros da espécie no conjunto de iscas e armadilhas e não com base no número de indivíduos (seg. Romero \& Jaffé 1989; Leal \& Lopes 1992) dividido pelo número total de iscas e armadilhas utilizadas multiplicado por 100. As espécies foram classificadas, segundo Silveira Neto et al. (1976), pela frequência de ocorrência como: acidental $(<25 \%)$, acessória $(>25<50 \%)$ e constante $(>50 \%)$.

\section{RESULTADOS}

Do total de 320 armadilhas de solo do tipo "pitfall" e iscas de sardinha distribuídas ao longo dos transectos, em 247 $(77,2 \%)$ foram encontradas formigas, sendo que o número de 


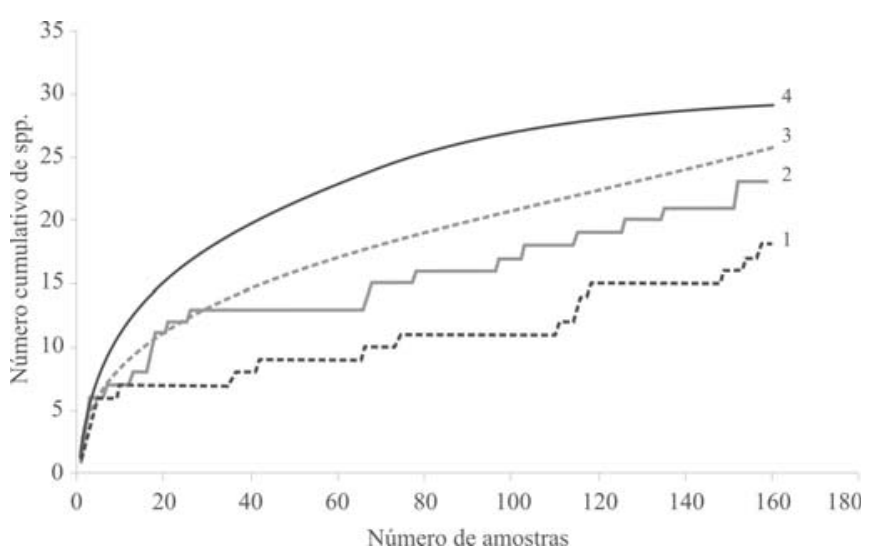

Fig. 1. Curvas cumulativas dos números de espécies observadas (Linha $1=$ iscas de sardinha e Linha $2=$ armadilhas de solo) e curvas de riqueza estimada de espécies de formigas em campo nativo no Planalto das Araucárias, Cambará do Sul, RS. (Linha 4= armadilhas de solo e linha $3=$ iscas de sardinha). Método não-paramétrico Jackknife de primeira ordem empregando-se 100 aleatorizações (EstimateS).

espécies por armadilha variou de uma a cinco e nas iscas de uma a três. Considerando as coletas manuais e as realizadas com armadilhas e iscas, foram coletadas 32 espécies de formigas epígeas, distribuídas em 16 gêneros, 12 tribos e cinco subfamílias (Tabela I). A subfamília Myrmicinae apresentou a maior riqueza de espécies ( $S=20)$, seguida por Formicinae $(S=7)$, Ponerinae ( $\mathrm{S}=3$ ), Dolichoderinae $(\mathrm{S}=2)$ e Ecitoninae $(\mathrm{S}=1)$. Os gêneros Camponotus (Formicinae), Solenopsis e Pheidole (Myrmicinae) foram os que apresentaram o maior número de táxons, os dois primeiros com quatro e o último com nove.

Comparando-se as riquezas observadas $\left(\mathrm{S}_{\text {obs }}\right)$ com os valores da riqueza estimada $\left(\mathrm{S}_{\text {est }}\right)$ tanto para as armadilhas $\left(\mathrm{S}_{\mathrm{est}}=28,96\right)$ como para as iscas $\left(\mathrm{S}_{\mathrm{est}}=25,95\right)$, os números de espécies coletadas pelas armadilhas e pelas iscas representaram, respectivamente, 79,4 e 69,4\% da comunidade total estimada (Figura 1). Das 28 espécies de formigas coletadas pelas armadilhas de solo e iscas de sardinha, 27 (96,4\%) foram classificadas como acidentais quanto ao padrão de ocorrência e somente Pheidole sp.3 (3,6\%) foi categorizada como acessória.

\section{DISCUSSÃO}

A riqueza de formigas epígeas registrada em ambiente de campo nativo na região do Planalto das Araucárias foi muito maior do que a encontrada em outra área de campo localizada a cerca de $65 \mathrm{~km}$ no entorno da Floresta Nacional de São Francisco de Paula (FLONA - SFP) por Diehl et al. (2005a) que registraram apenas três espécies, das quais somente Camponotus fastigatus Roger, 1873 não foi coletada em Cambará do Sul. Esta diferença certamente decorre do maior esforço amostral empregado e por terem sido utilizadas armadilhas de solo tipo pitfall e feitas coletas diretas, além das iscas de sardinha, que foi a única técnica de coleta empregada no entorno da FLONA-SFP. As armadilhas pitfall e as iscas de sardinha são técnicas que fornecem dados quantitativos que podem ser usados para se estabelecer a abundância das espécies, estimar a riqueza local e fazer comparações com dados de outras áreas e regiões. Por sua vez, a coleta manual fornece apenas dados qualitativos, porém é muito importante em levantamentos faunísticos. O ideal é associar técnicas de coleta quantitativas com qualitativas (Romero \& Jaffé 1989; Bestelmeyer et al. 2000). No entanto, o uso de uma ou mais técnicas vai depender do ambiente em questão, do tempo disponível para a realização das coletas e do número de pessoas envolvidas. Assim, o extrator de Winckler não pode ser usado pois é específico para coletas em serrapilheira, fazendo que apenas coletas diretas, iscas de sardinha e com armadilhas tipo pitfall sejam as técnicas a serem empregadas para formigas epígeas em áreas de campos. Quanto ao padrão de ocorrência, a grande maioria $(96,4 \%)$ das espécies foi categorizada como acidental e apenas uma como ocasional possivelmente pelas condições climáticas subtropicais da região e não como decorrência das técnicas de coleta.

O número de espécies coletadas, dentre outros fatores, reflete a complexidade estrutural da vegetação. Assim, as diferentes fisionomias dos campos nativos (limpo ou seco, rupestre e turfoso) associadas às manchas esparsas de mata de araucária devem ter contribuído para o aumento da riqueza de espécies, pois proporcionam uma maior diversidade de sítios de nidificação, além de ampliar a área de forrageamento das espécies (Hölldobler \& Wilson 1990).

Nos campos do Planalto das Araucárias a riqueza de espécies foi menor que em outras regiões do Rio Grande do Sul: 60 espécies de formigas em três ambientes (mata nativa, barreira pedregosa e areias da orla) no Parque Estadual de Itapuã (Diehl et al. 2005b), 49 em povoamentos de Eucalyptus spp. em áreas de restinga (Fonseca \& Diehl 2004) e 45 em áreas de minas de cobre (Diehl et al. 2004). Fica claro que o extremo sul do Brasil, por estar mais afastado da zona equatorial, apresenta uma riqueza de espécies menor do que nas zonas tropicais (Kuznezov 1957; Ward 2000), sendo que, em Manaus, por exemplo, foram encontradas mais de 300 espécies de formigas (Benson \& Harada 1988), enquanto no cerrado foram registradas 333 espécies (Silvestre et al. 2003).

O clima subtropical da região, acompanhado por invernos rigorosos, certamente também é um fator determinante do reduzido número de espécies. Os ambientes tropicais são altamente produtivos e, portanto, podem abrigar maior número de espécies de formigas. O fato destes insetos serem termofílicos também favorece a sua ocorrência em ambientes quentes. Nas zonas tropicais a riqueza de espécies arborícolas é alta, enquanto que em regiões mais frias, proporcionalmente, a maior riqueza é de espécies que nidificam no solo em áreas abertas e não sombreadas, como campos e savanas, com ninhos expostos ao sol ou em ninhos subterrâneos, onde a temperatura pode ser controlada pelas próprias formigas (Kaspari 2000). Baixas temperaturas associadas à ambientes sombrios têm um forte efeito estressante sobre elas, mas esses efeitos podem ser moderados nos ambientes frios com grande incidência de luz solar (Andersen 2000), o que pode justificar 
Tabela I. Padrões de ocorrência (PO) dos táxons de formigas coletados em ambiente de campo nativo na região do Planalto das Araucárias, Cambará do Sul, RS com armadilhas "pitfall" (A), iscas de sardinha (I) e coleta manual (M). N= número de iscas e armadilhas contendo formigas; FR=Freqüência relativa na amostragem $\left(=\mathrm{N}^{*} 100 / 320\right), 320$ sendo o número total de pontos coletados.

\begin{tabular}{|c|c|c|c|c|c|c|c|c|}
\hline \multirow{2}{*}{$\begin{array}{l}\text { Subfamília } \\
\text { Dolichoderinae }\end{array}$} & \multirow{2}{*}{$\begin{array}{l}\text { Tribo } \\
\text { Tapinomini }\end{array}$} & \multirow{2}{*}{$\begin{array}{l}\text { Táxon } \\
\text { Linepithema humile Mayr, } 1868\end{array}$} & \multicolumn{3}{|c|}{ Técnica } & \multirow{2}{*}{$\frac{\mathrm{N}}{13}$} & \multirow{2}{*}{$\begin{array}{l}\text { FR } \\
4,1\end{array}$} & \multirow{2}{*}{$\frac{\text { PO }}{\text { Acidental }}$} \\
\hline & & & $\mathrm{A}$ & I & $\mathrm{M}$ & & & \\
\hline & & & A & - & - & 1 & 0,3 & Acidental \\
\hline Ecitoninae & Ecitonini & Neivamyrmex sp. & - & - & M & - & - & - \\
\hline \multirow[t]{7}{*}{ Formicinae } & Camponotini & Camponotus (Myrmobrachys) mus Roger, 1863 & - & $\mathrm{I}$ & - & 1 & 0,3 & Acidental \\
\hline & & Camponotus (Myrmobrachys) crassus Mayr, 1862 & A & I & M & 34 & 10,6 & Acidental \\
\hline & & Camponotus (Myrmothrix) rufipes (Fabricius, 1775) & - & - & M & - & - & - \\
\hline & & Camponotus sp. 16 & - & - & M & - & - & - \\
\hline & Myrmelachistini & Brachymyrmex sp. 2 & $\mathrm{~A}$ & I & - & 10 & 3,1 & Acidental \\
\hline & Plagiolepidini & Acropyga (Rhizomyrma) goeldii Forel, 1893 & A & - & M & 1 & 0,3 & Acidental \\
\hline & Prenolepidini & Paratrechina sp. 3 & A & - & - & 1 & 0,3 & Acidental \\
\hline \multirow[t]{19}{*}{ Myrmicinae } & Attini & Acromyrmex crassispinus Forel, 1909 & A & - & - & 3 & 0,9 & Acidental \\
\hline & & Cyphomyrmex (gr. rimosus) sp. 1 & $\mathrm{~A}$ & - & - & 1 & 0,3 & Acidental \\
\hline & & Cyphomyrmex (gr. strigatus) sp. & A & - & - & 1 & 0,3 & Acidental \\
\hline & & Trachymyrmex sp. 1 & A & - & - & 1 & 0,3 & Acidental \\
\hline & Blepharidattini & Wasmannia sp. 1 & A & - & M & 1 & 0,3 & Acidental \\
\hline & Crematogastrini & Crematogaster sp. 1 & - & I & - & 1 & 0,3 & Acidental \\
\hline & Pheidolini & Pheidole (gr. fallax) sp. & A & I & - & 7 & 2,2 & Acidental \\
\hline & & Pheidole sp. 2 & A & I & M & 3 & 0,9 & Acidental \\
\hline & & Pheidole sp. 3 & A & I & M & 129 & 40,3 & Acessória \\
\hline & & Pheidole sp. 5 & A & $\mathrm{I}$ & - & 9 & 2,8 & Acidental \\
\hline & & Pheidole sp. 6 & $\mathrm{~A}$ & I & M & 51 & 15,9 & Acidental \\
\hline & & Pheidole sp. 7 & A & - & - & 2 & 0,6 & Acidental \\
\hline & & Pheidole sp. 15 & $\mathrm{~A}$ & $\mathrm{I}$ & - & 24 & 7,5 & Acidental \\
\hline & & Pheidole sp. 21 & A & - & - & 1 & 0,3 & Acidental \\
\hline & & Pheidole sp. 24 & A & I & - & 20 & 6,2 & Acidental \\
\hline & Solenopsidini & Solenopsis (Diplorhoptrum) sp. & A & $\mathrm{I}$ & - & 4 & 1,2 & Acidental \\
\hline & & Solenopsis invicta Buren, 1972 & A & I & - & 21 & 6,6 & Acidental \\
\hline & & Solenopsis sp. 2 & - & I & M & 1 & 0,3 & Acidental \\
\hline & & Solenopsis sp. 3 & - & I & - & 1 & 0,3 & Acidental \\
\hline \multirow[t]{3}{*}{ Ponerinae } & Ponerini & Hypoponera foreli (Mayr, 1887) & - & I & - & 1 & 0,3 & Acidental \\
\hline & & Hypoponera sp. 1 & - & - & M & - & - & - \\
\hline & & Pachycondyla striata Fr. Smith, 1858 & A & I & - & 15 & 4,7 & Acidental \\
\hline 5 & 12 & 32 & 23 & 18 & 12 & & & \\
\hline
\end{tabular}

a riqueza de espécies encontrada nos campos do Planalto das Araucárias.

Segundo Lattke (2003) são conhecidos, para as regiões da mata Atlântica, floresta de Araucaria, pampa e chaco, 28 gêneros de formigas com espécies endêmicas. Oito destes gêneros, Acromyrmex, Crematogaster, Cyphomyrmex, Hypoponera, Neivamyrmex, Pachycondyla, Pheidole e Trachymyrmex, foram registrados no Planalto das Araucárias e também em outras regiões do estado, embora nenhum dos táxons identificados ao nível específico nestes campos seja endêmico.

Da tribo Attini, foi coletada apenas uma espécie do gênero Acromyrmex (A. crassispinus) espécie bastante comum em outras regiões do Rio Grande do Sul (Mayhé-Nunes \& DiehlFleig 1994). Além desta, na FLONA-SFP, foram coletadas mais outras três espécies de Acromyrmex. Por outro lado, da mesma tribo, os gêneros Trachymyrmex e Cyphomyrmex estiveram representados por uma e duas espécies, respectivamente, enquanto que não foram registrados em manchas de floresta ombrófila mista na FLONA-SFP e na área de campo no seu entorno (Diehl et al. 2005a).
Os gêneros Pheidole (Myrmicinae) e Camponotus (Formicinae) são cosmopolitas e de ampla distribuição geográfica. Além de hiperdiversos, são dominantes tanto em número de operárias e de colônias como em biomassa, sendo frequentes nos mais diversos habitats (Wilson 1976, 2003). Por sua vez, Crematogaster (Myrmicinae) é um gênero que reúne um grande número de espécies tropicais, muitas das quais arborícolas (Andersen 2000), outras são habitantes de solo ou de serrapilheira, enquanto algumas mantêm associações com plantas ou com outras espécies de formigas (Fernández 2003).

As espécies dos gêneros Hypoponera, Neivamyrmex e Pachycondyla são predadoras, alimentando-se quase que exclusivamente de artrópodes de solo. O gênero Neivamyrmex, com uma dieta taxonomicamente limitada, reúne algumas espécies predadoras especializadas em caçar outras formigas, mas também cupins e outros insetos (Fowler et al. 1991; Kaspari 2000, 2003), o que faz com que necessitem de amplas áreas de forrageamento para suprir seus requisitos nutricionais, pois são predadoras muito eficientes e podem arrasar com a fauna 
local, obrigando a colônia a migrar para novos ambientes (Hölldobler \& Wilson 1990).

Algumas espécies, como Linepithema humile (Mayr) e Solenopsis invicta Buren, apresentam ninhos múltiplos com diversas rainhas e frequentemente dominam os habitats que invadem, graças a seu grande potencial de crescimento e extensão espacial (Kaspari 2003). Há relatos destas espécies causando grandes impactos nos ambientes que invadem (para revisão, veja Williams et al. 2003; Diehl-Fleig 2006), porém nos campos do Planalto das Araucárias não foram encontradas em altas densidades. Como não foram encontradas espécies nativas com características competidoras altas para dificultar a expansão daquelas espécies, especialmente de L. humile, pode-se supor que as invasões sejam recentes.

Com este levantamento pode ser feito o primeiro registro para o Rio Grande do Sul de Acropyga (Rhizomyrma) goeldii, que não havia sido citada nem por Kempf (1972) nem por Brandão (1991). O gênero Acropyga possui ampla distribuição, geográfica, mas apenas o subgênero Rhizomyrma está registrado para o Novo Mundo (Weber 1944). São formigas subterrâneas com cerca de dois milímetros de comprimento, com hábitos crípticos e, geralmente, nidificando sobre raízes (Delabie et al. 1991; Johnson et al. 2001). Algumas espécies apresentam associações obrigatórias com vários gêneros de Pseudococcidae hipogéicos da subfamília Rhizoecinae que ficam nas raízes de espécies arbóreas, especialmente, do cacau e café (Delabie et al. 1991; Johnson et al. 2001; Delabie \& Fernández 2003; Fernández 2003).

O padrão de ocorrência da grande maioria das espécies foi muito semelhante aos padrões esperados para a região Tropical com estações bem definidas, apesar do Planalto das Araucárias estar em altitude elevada na região Subtropical, caracterizada por invernos rigorosos e poucos períodos de altas temperaturas. Por outro lado, o ambiente heterogêneo, evidenciado pelas diferentes fisionomias de campo entremeadas com exemplares de A. angustifolia e de florestas de galeria no entorno e entre as áreas estudadas deve estar favorecendo a coexistência de várias espécies de formigas com amplitudes ecológicas maiores devido à diversidade das fontes alimentares exploradas. Apesar da crescente pressão antrópica das atividades agrosilvopastoris que essas áreas vêm sofrendo nos últimos anos, elas ainda parecem estar relativamente preservadas. Cabe ressaltar que uma das espécies identificadas ocorreu exclusivamente nesses campos. Assim, os resultados deste trabalho contribuem para o conhecimento da diversidade de formigas no Rio Grande do Sul e pode fornecer importantes informações para futuros planos de manejo e conservação destas áreas.

Agradecimentos. CAPES pela concessão da bolsa de mestrado da autora sênior; IBAMA por disponibilizar alojamento e pela autorização para coletas no Parque Nacional dos Aparados da Serra; Sr. Claudio Prestes por permitir coletas em sua propriedade; Maria Virgínia Petry pelo convite para integrar a equipe do Projeto Fogo; agradecemos também aos bolsistas e estagiários pelo auxílio nas coletas e triagem do material, e a A. J. Mayhé-Nunes, J. H. C. Delabie e a R. Feitosa pela identificação de algumas espécies.

\section{REFERÊNCIAS}

Agosti, D. \& N. F. Johnson. 2003. La nueva taxonomía de hormigas, p. 45-48. In: F. Fernández (ed.). Introducción a las hormigas de la región Neotropical. Bogotá, Colômbia, Instituto de Investigación de Recursos Biológicos Alexander von Humboldt, xxv +424 p.

Alonso, L. E. \& D. Agosti. 2000. Biodiversity studies, monitoring, and ants: an overview, p. 1-8. In: D. Agosti; J. D. Majer; L. E. Alonso \& T. R. Schultz (eds.). Ants: Standard methods for measuring and monitoring biodiversity. Washington, Smithsonian Institution Press, xix +280 p.

Andersen, A. N. 1987. Ant community organization and environmental assessment, p. 43-52. In: J. D. Majer (ed.). The role of invertebrates in conservation and biological survey. Perth, Western Australian, Department of Conservation and Land Management Report, $121 \mathrm{p}$.

Andersen, A. N. 2000. A global ecology of rainforest ants: functional groups in relation to environmental stress and disturbance. p. 2534. In: D. Agosti; J. D. Majer; L. E. Alonso \& T. R. Schultz (eds.). Ants: Standard methods for measuring and monitoring biodiversity. Washington, Smithsonian Institution Press, xix + $280 \mathrm{p}$.

Bestelmeyer, B. T.; D. Agosti; L. E. Alonso; C. R. F. Brandão; W. L. Brown Jr.; J. H. C. Delabie \& R. Silvestre. 2000. Field techniques for the study of ground-dwelling ants: an overview, description, and evaluation, p. 122-144. In: D. Agosti, J. D. Majer, L. E. Alonso \& T. R. Schultz (eds.). Ants: Standard methods for measuring and monitoring biodiversity. Washington, Smithsonian Institution Press, xix +280 p.

Benson, W. W. \& A. Y. Harada. 1988. Local diversity of tropical temperate ant faunas (Hymenoptera: Formicidae). Acta Amazonica 18: $275-289$.

Bolton, B. 1994. Identification guide to the ant genera of the world. Massachussets, Harvard University Press, 222 p.

Bolton, B. 2003. Synopsis and classification of Formicidae. Florida, Memoirs of the American Entomological Institute, 370 p.

Bonnet, A. \& B. C. Lopes. 1993. Formigas de dunas e restingas da praia da Joaquina, Ilha de Santa Catarina, SC (Insecta: Hymenoptera). Biotemas 6: 107-114.

Brandão, C. R. 1991. Adendo ao catálogo abreviado das formigas da Região Neotropical (Hymenoptera: Formicidae). Revista Brasileira de Entomologia 35: 319-412.

Bristot, A. 2001. Planalto das Araucárias - um ecossistema em perigo de extinção? Agroecologia e Desenvolvimento Rural Sustentável 4: 24-31.

Colwell, R. K. 1997. EstimateS: estatistical estimation of species richness and shared species from samples. Version 5.0.1. <http://viceroy.eeb.uconn.edu/ estimate>.

Delabie, J. H. C. \& F. Fernández. 2003. Relaciones entre hormigas y "homópteros" (Hemiptera: Sternorrhynca y Auchenorrhynca), p. 181-197. In: F. Fernández (ed.). Introducción a las hormigas de la región Neotropical. Bogotá, Colômbia, Instituto de Investigación de Recursos Biológicos Alexander von Humboldt, xxv $+424 \mathrm{p}$

Delabie, J. H. C.; J. E. Mantovani \& C. Mauricio. 1991. Observações sobre a biologia de duas espécies de Acropyga (Formicidae, Formicinae, Plagiolepidini) associadas à rizosfera do cacaueiro. Revista Brasileira de Biologia 51: 185-192.

Delabie, J. H. C.; B. L. Fisher; J. D. Majer \& I. W. Wright. 2000. Sampling effort and choice of methods, p. 145-154. In: D. Agosti, J. D. Majer, L. E. Alonso \& T. R. Schultz (eds.). Ants: Standard methods for measuring and monitoring biodiversity. Washington, Smithsonian Institution Press, xix +280 p.

Diehl, E.; C. E. D. Sanhudo \& Ed. Diehl-Fleig. 2004. Ground-dwelling ant fauna of sites with high levels of copper. Brazilian Journal of Biology 64: 33-39.

Diehl, E.; D. F. Florencio; F. A. Schmidt \& L. V. A. Menzel. 2005a. Riqueza e composição das comunidades de formigas e de térmitas na 
Floresta Nacional de São Francisco de Paula (FLONA-SFP), RS. Acta Biologica Leopoldensia 27: 99-106.

Diehl, E.; F. Sacchett \& E. Z. Albuquerque. 2005b. Riqueza de formigas de solo na praia da Pedreira, Parque Estadual de Itapuã, Viamão, RS. Revista Brasileira de Entomologia 49: 552-556.

Diehl-Fleig, Ed. 2006. Formigas invasoras: o caso da formiga Argentina Linepithema humile (Mayr 1868). Acta Biologica Leopoldensia 28: 5-9.

Fernández, F. 2003. Subfamília Formicidae, p. 299-306. In: F. Fernández (ed.). Introducción a las hormigas de la región Neotropical. Bogotá, Colômbia, Instituto de Investigación de Recursos Biológicos Alexander von Humboldt, xxv +424 p.

Fitkau, E. J. \& H. Klinge. 1973. On biomass and trophic structure of the Central Amazonian Rain Forest ecosystem. Biotropica 5: 214.

Fonseca, R. C. \& E. Diehl. 2004. Riqueza de formigas (Hymenoptera, Formicidae) epigéicas em povoamentos de Eucalyptus spp. (Myrtaceae) de diferentes idades no Rio Grande do Sul, Brasil. Revista Brasileira de Entomologia 48: $95-100$.

Fowler, H. G.; L. C. Forti; C. R. F. Brandão; J. H. C. Delabie \& H. L. Vasconcelos. 1991. Ecologia nutricional de formigas, p. 131-223. In: A. R. Panizzi \& J. R. P. Parra (eds.). Ecologia nutricional de insetos e suas aplicações no manejo de pragas. São Paulo, Editora Manole, $359 \mathrm{p}$.

Hölldobler, B. \& E. O. Wilson. 1990. The Ants. Massachussets, The Belknap Press of Harvard University Press, xii +732 p.

Jaffé, K. C. 1993. El mundo de las hormigas. Maracay, Venezuela, Universidad Simon Bolívar, 187 p.

Johnson, C.; D. Agosti; J. H. Delabie; K. Dumpert; D. J. Williams; M. Von Tschirnhaus \& U. Maschwitz. 2001. Acropyga and Azteca ants (Hymenoptera: Formicidae) with scale insects (Sternorrhyncha: Coccoidea): 20 million years of intimate symbiosis. American Museum Novitates 3335: 1-18.

Kaspari, M. 2000. A primer on ant ecology, p. 9-24. In: D. Agosti, J. D. Majer, L. E. Alonso \& T. R. Schultz (eds.). Ants: Standard methods for measuring and monitoring biodiversity. Washington, Smithsonian Institution Press, xix +280 p.

Kaspari, M. 2003. Introducción a la ecologia de las hormigas, p. 97112. In: F. Fernández (ed.). Introducción a las hormigas de la región Neotropical. Bogotá, Instituto de Investigación de Recursos Biológicos Alexander von Humboldt, xxv +424 p.

Kempf, W. W. 1972. Catálogo abreviado das formigas da Região Neotropical (Hymenoptera: Formicidae). Studia Entomologica 15: $1-345$.

Kusnezov, N. 1957. Numbers of species of ants in faunae of different latitudes. Evolution 11: 298-299.

Lattke, J. E. 2003. Biogeografía de las hormigas neotropicales, p. 6585. In: F. Fernández (ed.). Introducción a las hormigas de la región Neotropical. Bogotá, Colômbia, Instituto de Investigación de Recursos Biológicos Alexander von Humboldt, xxv +424 p.

Leal, I. N. \& B. C. Lopes. 1992. Estrutura das comunidades de formigas (Hymenoptera: Formicidae) de solo e vegetação no Morro da Lagoa da Conceição, Ilha de Santa Catarina, SC. Biotemas 5: 107-122.

Lobry de Bruyn, L. A. 1999. Ants as bioindicators of soil function in rural environments. Agriculture, Ecosystems and Environment 74: 425-441.

Majer, J. D. 1983. Ants: bio-indicators of minesite rehabilitation, landuse, and land conservation. Environmental Management 7: 375383.

Majer, J. D. 1984. Ant return in rehabilited mines: an indicator of ecosystem resilience. Proceedings of the $4^{\text {th }}$ International
Conference on Mediterranean Ecosystems: 105-106.

Majer, J. D.; J. E. Day; E. D. Kabay \& W. S. Perriman. 1984. Recolonization by ants in bauxite mines rehabilited by a number of different methods. Journal of Applied Ecology 21: 355-375.

Matos, J. Z.; C. N. Yamanaka; T. T. Castellani \& B. C. Lopes. 1994. Comparação da fauna de formigas de solo em áreas de plantio de Pinnus elliotii, com diferentes graus de complexidade estrutural (Florianópolis, SC). Biotemas 7: 57-64.

Mayhé-Nunes, A. J. \& E. Diehl-Fleig. 1994. Distribuição de Acromyrmex (Hymenoptera: Formicidae) no Rio Grande do Sul. Acta Biologica Leopoldensia 16: 115-118.

Oliveira, M. A.; T. M. C. Della-Lucia; M. S. Araújo \& A. P. Cruz. 1996. A fauna de formigas em povoamentos de eucalipto e mata nativa no estado do Amapá. Acta Amazonica 25: 117-126.

Plano de Manejo do Parque Nacional dos Aparados da Serra. 2003. Cambará do Sul, RS. (www.members.tripod.com/cambara_do_sul/ cidade.html). Acessado em julho 2005.

Rambo, B. 1956. A fisionomia do Rio Grande do Sul. Porto Alegre, Selbach De Selbach, 456 p.

Romero, H. \& K. Jaffé. 1989. A comparison of methods for sampling ants (Hymenoptera: Formicidae) in savannas. Biotropica 21: $348-$ 352.

Silveira Neto, S.; D. Barbin \& N. A. Villa Nova. 1976. Manual de Ecologia dos Insetos. São Paulo, Editora Agronômica Ceres, 419 p.

Silvestre, R. C. R. F. Brandão \& R. R. Silva. 2003. Grupos funcionales de hormigas: el caso de los grêmios del cerrado, p.113-148. In: F. Fernández (ed.). Introducción a las hormigas de la región Neotropical. Bogotá, Colômbia, Instituto de Investigación de Recursos Biológicos Alexander von Humboldt, xxv $+424 \mathrm{p}$.

Tavares, A. A.; P. C. Bispo \& A. C. S. Zanzini. 2001. Comunidades de formigas epigéicas (Hymenoptera, Formicidae) em áreas de Eucalyptus cloeziana $\mathrm{F}$. Muell. e de vegetação nativa numa região de cerrado. Revista Brasileira de Entomologia 45: 251-256.

Teixeira, M. B.; A. B. Coura Neto; U. Pastore \& A. R. L. Rangel Filho. 1986. Vegetação, p. 541-620. In: IBGE (ed.). Levantamento de recursos naturais. Rio de Janeiro, IBGE, $791 \mathrm{p}$.

Ward, P. S. 2000. Broad-scale patterns of diversity in leaf litter ant communities, p. 99-121. In: D. Agosti, J. D. Majer, L. E. Alonso \& T. R. Schultz (eds.). Ants: Standard methods for measuring and monitoring biodiversity. Washington, Smithsonian Institution Press, xix +280 p.

Weber, N. A. 1944. The Neotropical coccid-tending ants of the genus Acropyga Roger. Annals of the Entomological Society of America 37: 89-122.

Williams, D. F.; D. H. Oi; S. D. Porter; R. M. Pereira \& J. A. Briano 2003. Biological control of imported fire ants (Hymenoptera; Formicidae). American Entomologist 49: 150-163.

Wilson, E. O. 1976. Which are the most prevalent ant genera? Studia Entomologica 19: 187-200.

Wilson, E. O. 1997. A situação atual da diversidade biológica, p. 3-24. In: E. O. Wilson \& M. P. Frances (eds.). Biodiversidade. Rio de Janeiro, Nova Fronteira, $657 \mathrm{p}$.

Wilson, E. O. 2000. Foreword, p. xv-xvi. In: D. Agosti, J. D. Majer, L. E. Alonso \& T. R. Schultz (eds.). Ants: Standard methods for measuring and monitoring biodiversity. Washington, Smithsonian Institution Press, xix +280 p.

Wilson, E. O. 2003. La hiperdiversidad como fenomeno real: el caso de Pheidole. p. 363-370. In: F. Fernández (ed.). Introducción a las hormigas de la región Neotropical. Bogotá, Colômbia, Instituto de Investigación de Recursos Biológicos Alexander von Humboldt, $\mathrm{xxv}+424 \mathrm{p}$. 